\title{
5. PALM OIL CAROTENOIDS
}

\section{THE ISOLATION OF LIPOID PIGMENTS FROM A 'SHERBRO' PALM OIL}

\author{
BY ROBERT FERGUS HUNTER AND ALAN DALTON SCOTT \\ From the Central Technical Department, Lever Brothers and Unilever, Ltd., \\ Port Sunlight
}

(Received 25 November 1940)

The colour of red African palm oils is generally attributed in the main to the presence of carotene, a relatively high proportion of which is assumed to occur in the form of the optically active $\alpha$-isomer [Kuhn \& Brockmann, 1931]. Actually, qualitative chromatographic examination of the unsaponifiable matter of typical palm oils provides immediate evidence of the multiplicity of the coloured constituents exhibiting the usual carotenofid characteristics. It is to be expected that the nature of the carotenoids present in palm oils will vary to some extent with the district of their origin and their previous history. It therefore appeared to us that an examination of some typical palm oils might prove of interest from the point of view of identifying known carotenoids and throwing new light on the problem of their biogenesis; moreover, such investigations might furnish examples of other members of the group which have not previously been detected in nature. In this paper, which is to be regarded as of an introductory character, attention has been directed to an investigation of the carotenoids present in 'Sherbro' palm oil.

With the object of minimizing as far as possible any tendency for the carotenoids to undergo isomeric changes of the type recently described by Zechmeister \& Tuzson [1938], and by Carter \& IGillam [1939], the saponification of the oil was accomplished at as low a temperature as reasonably possible. Extraction with light petroleum and thereafter with methylated ether, followed by evaporation under reduced pressure in an atmosphere of $\mathrm{CO}_{2}$, furnished fractions which may be described for the sake of convenience as petrol-soluble unsaponifiable matter and ether-soluble unsaponifiable matter. The former of these, on chromatographic adsorption on a Tswett column of alumina, using a modified technique in which the separation is conducted in an atmosphere of $\mathrm{CO}_{2}$, gave a series of coloured zones which on elution and subsequent purification furnished beautifully crystalline specimens of $\alpha$ - and $\beta$-carotenes and carotenoids having the spectroscopic and other characteristics of $\gamma$-carotene and lycopene.

The physical properties of the specimens of $\alpha$ - and $\beta$-carotene so obtained indicate that these are almost certainly purer than any which have previously been described in the literature. The dextrorotation of $\alpha$-carotene in benzene for the sodium $\mathrm{D}$ line is recorded for the first time. Curiously enough, nearly all previous measurements have been made with respect to the less intense cadmium - line, for which the value $[\alpha]_{6435}=+385^{\circ}$ has been generally accepted. The value for the shorter wave-length of the sodium $D$ line corresponding to this cannot be deduced in the absence of an accurate knowledge of the rotatory dispersion of the 
compound, but the rotation of $+584^{\circ}$ for the helium line 5876, recorded by Smith \& Milner [1934] for a specimen melting some degrees lower than ours, is reasonably close to our determination of $+640^{\circ}$ for the sodium line 5893. Solutions of our specimens of $\alpha$ - and $\beta$-carotene in arachis oil which were submitted to Dr H. Wilkinson of our Nutrition Laboratory for biological test provided striking confirmation of Kuhn \& Brockmann's [1933, 2] 1:2 ratio for the potencies of $\alpha$ - and $\dot{\beta}$-carotene.

The lycopene zone of the chromatogram of the petrol-soluble unsaponifiable matter, on being eluted and rechromatographed on alumina, furnished upper and lower dark red zones containing lycopene and neolycopene respectively. The closeness of the absorption maxima of neolycopene and $\gamma$-carotene would at first sight appear confusing, were it not for the finer differences in adsorptive power which permit the separation of the carotene from the 'total lycopene' zone.

The $\gamma$-carotene zone of the original chromatogram, on elution and readsorp'tion on alumina, furnished a single main dark red zone containing $\gamma$-carotene. A lighter coloured fringe appeared below this on the second chromatogram, which gave a small quantity of an unidentified carotenoid having maxima of a considerably shorter wave-length than those of $\gamma$-carotene. In a subsequent experiment in which more thorough development of the chromatogram of the petrol-soluble unsaponifiable matter was obtained, the zone containing this new carotenoid appeared between $\gamma$ - and $\beta$-carotene. What appears to be neo- $\gamma$ carotene was obtained when a chromatogram from a concentrate of $\gamma$-carotene was very thoroughly developed with a mixture of benzene and light petroleum.

Evaporation of the percolate from the original chromatogram of the petrolsoluble unsaponifiable matter gave a small amount of hentriacontane, accompanied by a quantity of a high-boiling point yellow oil which has not been identified. It may be-noted that Carter et al. [1939] observed the presence of what appears to be a similar material, which was assumed to be of a terpene character, in algae. The ether-soluble unsaponifiable matter on being dissolved in petrol and partitioned with $90 \%$ methyl alcohol gave a hypophasic fraction which on chromatographic adsorption on alumina gave a coloured zone containing neolutein. The epiphasic fraction, which contained quantities of sterols, gave on chromatographic analysis three zones containing substances which showed vague but intense spectroscopic absorption. They gave the usual reaction with chloroformic antimony trichloride with sharply defined maxima in the $590 \mathrm{~m} \mu$ region and were most probably oxidized carotenoids.

The presence of $\gamma$-carotene and lycopene in addition to $\alpha$-and $\beta$-carotenes in this palm oil seems suggestive from the point of view of the biogenesis of these pigments. The photosynthesis of lycopene by dehydrogenation of the product of the benzoin condensation of two molecules of the aldehyde corresponding to phytol has already been suggested by Karrer et al. [1930]. That lycopene is the precursor of $\beta$-carotene seems to us, however, unlikely, since a ring-chain isomerization of this type finds no parallel in synthetic organic chemistry. If ring-chain prototropy were possible, the presenoe of the terminal gem-dimethyl group of lycopene would, on the basis of the modified strain theory of Ingold and Thorpe, be expected to promote five- rather than six-membered ring formation [cf. Ingold, 1921]. Application of the general expression for ring-chain prototropy

$$
(A)\left\langle_ { Y = Z } ^ { X [ \mathrm { H } ] } \rightleftharpoons ( A ) \left\langle_{Y-Z[\mathrm{H}]}^{X}\right.\right.
$$

to lycopene would lead to a cyclic isomeride quite different from $\beta$-carotene. 
It seems more likely therefore that lycopene, $\beta$-carotene and also $\gamma$-carotene are formed simultaneously from the same intermediate complex, the $\gamma$-isomer representing the cyclization of only one end of the hydrocarbon chain:

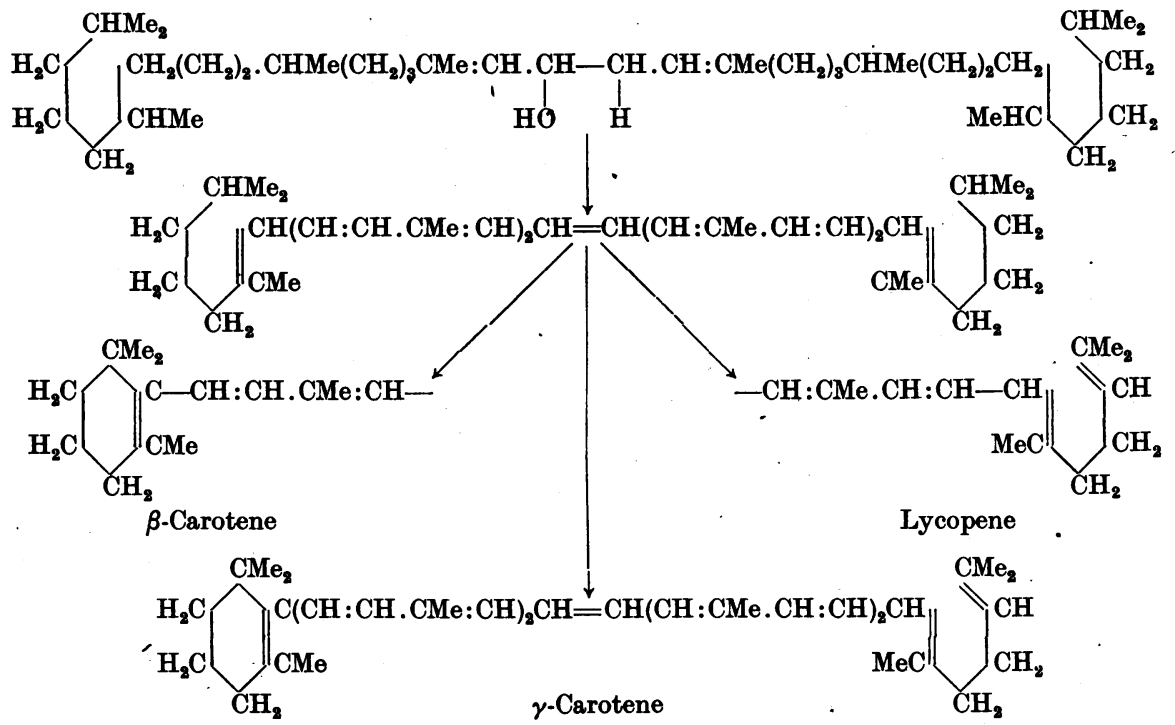

The appearance of $\alpha$-carotene may be explained as being due to asymmetric isomerization of part of the $\beta$-carotene. Such a change would be of the true three-carbon type:

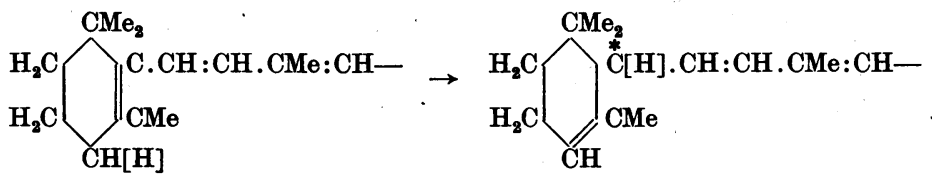

$$
\text { 1 }[\mathrm{H}]=\text { mobile hydrogen atom. }
$$

analogous to the racemization of $d$-benzhydrylidene- $\alpha$-phenylethylamine [Hsu et al. 1935]:

$$
\left.{ }_{\mathrm{Me}}^{\mathrm{Ph}}\right\rangle^{*}[\mathrm{H}] \cdot \mathrm{N}=\mathrm{C}\left\langle\left\langle_{\mathrm{Ph}}^{\mathrm{Ph}} \rightleftharpoons{ }_{\mathrm{Me}}^{\mathrm{Ph}}\right\rangle \mathrm{C}=\mathrm{N} \cdot \mathrm{C}[\mathrm{H}]\left\langle_{\mathrm{Ph}}^{\mathrm{Ph}}\right.\right.
$$

The latter change is concerned with the disappearance of optical activity due to mobility of the azomethine triad system $[\mathrm{H}] \mathrm{C} . \mathrm{N}: \mathrm{C} \rightleftharpoons \mathrm{C}: \mathrm{N}$. C $[\mathrm{H}]$, which is closely related to the three-carbon system [Ingold \& Shoppee, 1929]. The corresponding process of asymmetric synthesis evidently occurs in biochemical reactions in view of the frequent appearance of optical activity in natural products. A case of isomeric change involving movement of the centre of asymmetry without loss of optical activity, which is of some interest in this connexion, is furnished by the conversion of linalyl acetate into the correspondingly optically active $\alpha$-terpinyl ester [Stefan, 1898; Zeitschel, 1906; Ingold \& Wilson, 1933]:

Biochem. 1941, 35

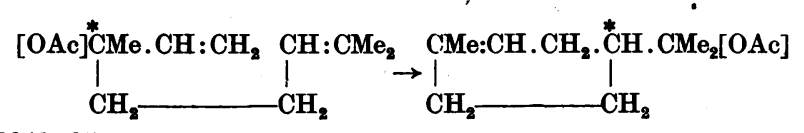


The isomeric change in this case is, however, of the anionotropic type and differs from the examples studied by Hsu et al. [1935] in that the migrating centre of asymmetry does not follow the route of the mobile group.

\section{Experimental}

The sample of 'Sherbro' palm oil used.for the following experiments was of recent importation and of good colour. The free fatty acid content determined by titration was $15 \%$, calculated as palmitic acid.

The oil (1500 g.) was saponified in two batches as follows. A solution of $\mathrm{KOH}$ (225 g.) in alcohol $\left(95 \%\right.$; $1300 \mathrm{ml}$.) was cooled to $35^{\circ}$ and the palm oil (750 g.) was gradually added with shaking. After a short time sufficient soap was formed to render the liquids miscible and light petroleum $(750 \mathrm{ml}$.$) and water (400 \mathrm{ml}$. were added to the mixture, which was heated to boiling and thereafter kept overnight at laboratory temperature. The mixture was heated under reflux for $1 \mathrm{hr}$. $\left(c a .45^{\circ}\right)$, cooled, diluted with alcohol $(95 \% ; 3500 \mathrm{ml}$.) and then with water to produce a final dilution to $15 \mathrm{l}$. The resulting soap solution was thrice extracted with light petroleum and the united extracts were washed with water until free from soap and alkali and dried over anhydrous $\mathrm{Na}_{2} \mathrm{SO}_{4}$. Evaporation under reduced pressure in an atmosphere of $\mathrm{CO}_{2}$ in an all-glass Quickfit apparatus furnished the petrol-soluble unsaponifiable matter which amounted to $5 \cdot 3 \mathrm{~g}$. This procedure, which was used in all evaporations to dryness described in this paper, enables the temperature to be kept below $50^{\circ}$.

The residual soap solution was further diluted and thrice extracted with methylated ether, the combined extracts being washed, dried and evaporated in $\mathrm{CO}_{2}$ as in the previous case. The ether-soluble unsaponifiable matter weighed $4 \cdot 1 \mathrm{~g}$.

The petrol-soluble unsaponifiable matter was resaponified by heating with a mixture of light petroleum $(330 \mathrm{ml}$.), alcohol $(670 \mathrm{ml}$.$) , benzene (20 \mathrm{ml}$.$) and$ $\mathrm{NaOH}(10 \mathrm{~g}$.) for $4 \mathrm{hr}$. The cooled mixture was twice extracted with light petroleum and the united extracts were washed, dried and evaporated as before. The residue so obtained was dissolved in light petroleum (ca. $400 \mathrm{ml}$.) containing benzene $(10 \mathrm{ml}$.), and the solution was passed down a Tswett column $(18 \mathrm{~mm}$. bore) of active alumina (British Drug Houses Ltd.) under slight $\mathrm{CO}_{2}$ pressure. The chromatogram was developed with a $10 \%$ solution of benzene in light petroleum when the following zones were obtained in order of decreasing adsorption: dark red brown (medium depth, zone 1), dark red (narrow, zone 2), dark orange red (narrow, zone 3), red orange (broad, zone 4) and very pale yellow (medium depth, zone 5). Each zone was separated and the organic material was eluted by alternate washings with acetone and ether and recovered by evaporation under reduced $\mathrm{CO}_{2}$ pressure.

Zone 1. The material extracted from this zone amounted to $1.56 \mathrm{~g}$.; a portion of it dissolved in alcohol $(95 \%)$ furnished a small quantity of a crystalline digitonide. This confirmed the expected presence of sterol, but the material was not further investigated. An attempt to crystallize the original material from light petroleum-methanol gave a sticky residue which showed broad but vague maxima at $480-485 \mathrm{~m} \mu$ and $455 \mathrm{~m} \mu$ in carbon disulphide. It gave the usual reaction with chloroformic antimony trichloride with a maximum at $590 \mathrm{~m} \mu$.

Zone 2. The extract from this zone furnished $0.28 \mathrm{~g}$. of a somewhat sticky solid which was dissolved in a small amount of benzene. This solution was diluted with light petroleum to give a mixture containing approximately $10 \%$ of benzene and adsorbed on a column of active alumina (British Drug Houses Ltd.). 
The chromatogram was developed with a similar mixture of benzene and light petroleum when four zones were obtained below a little oxidized material: dark red (broad, zone $2 a$ ), very dark red (narrow, zone $2 b$ ), extremely dark red (medium depth, zone $2 c$ ) and very dark red (narrow, zone $2 d$ ).

Zone $2 a$ (lycopene). The material extracted from this zone weighed $0 \cdot 15 \mathrm{~g}$. and showed definite maxima at 544 and $505 \mathrm{~m} \mu$ and a vague maximum at $481 \mathrm{~m} \mu$ in carbon disulphide. It gave a reaction with antimony trichloride with a maximum at $582 \mathrm{~m} \mu$. From a benzene-methanol solution of this a few $\mathrm{mg}$. of a red solid were obtained (M.P. ca. $147^{\circ}$ ) which showed maxima at 545, 507 and 474$475 \mathrm{~m} \mu$ in carbon disulphide and a maximum at $595 \mathrm{~m} \mu$ in the antimony trichloride reaction. It appears likely therefore that the displacement of the maximum to $582 \mathrm{~m} \mu$ in the antimony trichloride reaction with the crude material was due to some colourless impurity which does not affect the maxima of the direct colour spectrum.

Zone $2 b$ (lycopene, traces of neolycopene). The residue $(0.05 \mathrm{~g}$.) obtained from this could not be crystallized and showed maxima at 543-544, 505 and $478 \mathrm{~m} \mu$ (vague). It gave a broad maximum at $595 \mathrm{~m} \mu$ in the antimony trichloride reaction.

Zone $2 c$ (neolycopene). Evaporation of the extract furnished a red gum without any trace of crystalline material which showed maxima at 535, 496 and $469 \mathrm{~m} \mu$ (vague) in carbon disulphide. This material gave a broad maximum at $595 \mathrm{~m} \mu$ in the antimony trichloride reaction.

Zone $2 d$ ( $\gamma$-carotene). Evaporation of the extract from this zone gave $0.015 \mathrm{~g}$. of material. No crystals were obtained but portions of the residue showed maxima at 530, 495 and $466 \mathrm{~m} \mu$ (vague) in carbon disulphide. The antimony trichloride reaction gave a broad maximum at $590 \mathrm{~m} \mu$.

Zone 3 ( $\gamma$-carotene). This zone furnished $0.2 \mathrm{~g}$. of a somewhat sticky solid which was dissolved in a small amount of benzene; the solution was diluted with light petroleum and subjected to chromatographic separation on alumina. The chromatogram was developed with a $10 \%$ solution of benzene in light petroleum and formed a single main zone with a lighter-coloured fringe at the bottom. Élution of the main zone gave gummy material which showed bands at 529, 496 and $463 \mathrm{~m} \mu$ in carbon disulphide and a maximum at $595 \mathrm{~m} \mu$ in the antimony trichloride reaction. A solution of this in benzene-methanol gave a trace of solid which showed maxima at 530, 496 and $463 \mathrm{~m} \mu$ in carbon disulphide and a maximum at $595 \mathrm{~m} \mu$ in the antimony trichloride reaction. Kuhn \& Brockmann $[1933,2]$ record $533.5 \mathrm{~m} \mu$ for the first maximum of $\gamma$-carotene in carbon disulphide, but Carter et al. [1939] have drawn attention to the fact that the first maximum observed in a prism spectrophotometer tends to be situated some few $\mathbf{m} \mu$ further towards the shorter wave-length region than that seen with grating instruments.

Elution of the lower yellow fringe of the chromatogram gave a trace of material which showed bands at 486,458 and $426 \mathrm{~m} \mu$ in carbon disulphide and a maximum at $595 \mathrm{~m} \mu$ in the antimony trichloride reaction. In a later experiment with the same 'Sherbro' palm oil, this substance was observed between the $\gamma$-carotene and $\beta$-carotene zones in the large chromatogram of the petrol-soluble unsaponifiable matter. In this experiment a $\gamma$-carotene concentrate was obtained which was separated by chromatographic analysis into $\gamma$-carotene (maxima at 529, 495 and $465 \mathrm{~m} \mu$ in carbon disulphide and at 502, 473-474 and $449 \mathrm{~m} \mu$ in chloroform) and what we believe to be neo- $\gamma$-carotene (maxima at 520,488 and $455 \mathrm{~m} \mu$ in carbon disulphide).

Zone 4 ( $\alpha$-and $\beta$-carotenes). The mixture of carotenes obtained by elution of 
this large zone was dissolved in benzene (100 ml.). An aliquot portion of this diluted with chloroform gave an $E 1 \%$, value of 1710 at $458-459 \mathrm{~m} \mu$, indicating a carotene content of about $75 \%$ in the crude material. An alcoholic solution of the latter failed to give a digitonide, suggesting that the colourless impurity was not a sterol.

Portions of the benzene solution corresponding to approximately $0.15 \mathrm{~g}$. of carotene were diluted with light petroleum (9 vol.) and the solutions were passed down Tswett columns of Savory and Moore's 'Mayfair' alumina. The chromatograms were developed with a 25-30\% solution of benzene in light petroleum and the lighter-coloured $\alpha$-carotene zones were separated from the more strongly adsorbed $\beta$-isomer. The pigments were eluted with acetone and the crude carotenes were recovered in the usual way.

A solution of the $\beta$-carotene concentrate in acetone deposited a crystalline solid which was collected,-dissolved in a $20 \%$ solution of benzene in light petroleum and subjected to chromatographic separation on 'Mayfair' alumina for a second time. The central portion of the $\beta$-carotene zone was separated and eluted, and the recovered pigment was crystallized as rapidly as possible from acetone to prevent any isomerization. The $\beta$-carotene thus obtained formed beautiful glistening purple red plates, M.P. $184 \cdot 5^{\circ}$ (uncorr.; open melting point tube). A solution of the crystals in chloroform had an $E 1 \%$. value of 2310 at $463 \mathrm{~m} \mu$, which is appreciably higher than the value recorded by Gillam [1935] for $\beta$-carotene in this solvent. The antimony trichloride reaction showed a maximum at $592 \mathrm{~m} \mu$ with an $E 1 \%$ colue of 423 at the peak of the intensity. It has been observed that the purest specimens of $\beta$-carotene which we have obtained in this and other investigations give a rapidly fading maximum in the blue solution obtained in the antimony trichloride reaction.

A solution of the $\alpha$-carotene concentrate in acetone furnished a crystalline solid which was dissolved in benzene and the solution was diluted with light petroleum and passed down a column of 'Mayfair' alumina. The chromatogram was developed with $30 \%$ benzene in light petroleum and the main zone of $\alpha$-carotene was eluted. The $\alpha$-carotene recovered in the usual way was crystallized from acetone within $1 \frac{1}{2} \mathrm{hr}$., when it was obtained in glistening red-brown crystals, M.P. $187-187 \cdot 5^{\circ}$ (uncorr.; open melting point tube). This specimen showed maxima at 511, 479 and $450 \mathrm{~m} \mu$ in carbon disulphide, at 489,458 and $430 \mathrm{~m} \mu$ in chloroform, and at 489, 458 and $431 \mathrm{~m} \mu$ in benzene. The antimony trichloride reaction showed a main band at $495 \mathrm{~m} \mu$, a weak band at $650 \mathrm{~m} \mu$, an inflexion at $527 \mathrm{~m} \mu$ and a weak inflexion at $605 \mathrm{~m} \mu$. The $E_{1}^{1 \%} \mathrm{~cm}$. value for the maximum at $458 \mathrm{~m} \mu$ in chloroform was 2430 .

The optical rotation was determined in benzene solution, using a Hilger polarimeter M. 323 and a G.E.C. Osira sodium lamp as a source of monochromatic light. The instrument was adjusted for a half-shadow angle of $4^{\circ}$. Two determinations, adopting in each case the mean of ten readings, gave the following results:

$$
\begin{aligned}
& {[\alpha]_{D}=\frac{0.162 \times 100^{\circ}}{2 \times 0.0127}=+638^{\circ},} \\
& \dot{[} \alpha]_{D}=\frac{0.276 \times 100^{\circ}}{2 \times 0.02118}=+651^{\circ} .
\end{aligned}
$$

A similar G.E.C. Osira cadmium lamp gave totally inadequate illumination for use with any carotene solution of sufficient concentration to possess detectable rotation.

Zone 5. Elution of this gave a yellow oil (1.6 g.). A solution of this in alcohol $(95 \%)$ gave no precipitate with digitonin. An acetone-light petroleum 
solution of the oil furnished a small quantity of hentriacontane, which separated from acetone as a waxy white solid, M.P. $64-65^{\circ}$.

The ether-soluble unsaponifiable matter from the original saponification was dissolved in light petroleum (250 ml.) and the solution was extracted ten times with $90 \%$ methyl alcohol (total of $500 \mathrm{ml}$.). The petrol solution was dried over anhydrous $\mathrm{Na}_{2} \mathrm{SO}_{4}$ and evaporated to dryness at a temperature below $50^{\circ}$; the residue was adsorbed on a column of 'Birlec' alumina. The chromatogram developed by a $15 \%$ solution of benzene in light petroleum formed three zones: dark red (broad; zone 6), orange red (medium depth; zone 7) and yellow ochre (narrow; zone 8).

Zone 6. An acetone extract from this furnished crystalline sterols and an alcoholic solution of these gave a copious precipitate of digitonides, neither of which were investigated. The colouring matter present showed a broad and vague maximum at about $485-490 \mathrm{~m} \mu$ in carbon disulphide and gave the usual reaction with chloroformic antimony trichloride with a maximum at $590 \mathrm{~m} \mu$.

Zone \%. This furnished a gummy material which showed a similar vague maximum in the $485 \mathrm{~m} \mu$ region and gave a band at $588 \mathrm{~m} \mu$ in the antimony trichloride reaction.

Zone 8 . This also failed to furnish a crystalline pigment and gave a gum which showed maxima at 505 and $480 \mathrm{~m} \mu$ in carbon disulphide. The antimony trichloride reaction showed a transient band at $590 \mathrm{~m} \mu$, a weak but persistent band at $495 \mathrm{~m} \mu$ and a slight maximum at $650 \mathrm{~m} \mu$ which increased during observation.

The hypophasic portion of the ether-soluble unsaponifiable matter recovered from $90 \%$ methyl alcohol was subjected to chromatographic adsorption on a column of 'Birlec' alumina. The chromatogram, although developed with a $35 \%$ solution of benzene in light petroleum, furnished only a single orange red zone (medium depth; zone 9 ).

Zone 9 (neolutein). The crude carotenoid obtained from this zone formed a gum which gave a negative reaction with alcoholic digitonin. It showed maxima at 501 and $473 \mathrm{~m} \mu$ in carbon disulphide and a band at $595 \mathrm{~m} \mu$ in the antimony trichloride reaction.

\section{SUMMARY}

1. Examination of the unsaponifiable matter of 'Sherbro' palm oil revealed the presence of $\alpha$-, $\beta$ - and $\gamma$-carotenes, accompanied by lycopene, neolycopene, neolutein and what appears to be neo- $\gamma$-carotene. A new carotenoid which is adsorbed at a position intermediate between $\gamma$ - and $\beta$-carotenes on the chromatogram was also observed.

2. The physical properties of the specimens of $\alpha$ - and $\beta$-carotenes so obtained indicate that these are probably somewhat purer than any which have been described in the literature.

3. The biogenesis of carotenoids is discussed in the light of the phenomena of ring-chain tautomerism and prototropic change involving asymmetric isomerization. It is suggested that lycopene, $\beta$-carotene and also $\gamma$-carotene are formed concurrently from the same intermediate complex and that $\alpha$-carotene may arise from subsequent isomerization of the $\beta$-compound.

We wish to thank the directors of Lever Brothers and Unilever Ltd. for permission to publish these results. We also wish to express our thanks to Dr J. R. Edisbury for his help in connexion with some of the spectroscopic measurements. 


\section{REFERENCES}

Carter \& Gillam (1939). Biochem. J. 33, 1325.

Heilbron \& Lythgoe (1939). Proc. roy. Soc. B, 128, 82.

Gillam (1935). Biochem. J. 29, 1831.

Hsu, Ingold \& Wilson (1935). J. chem. Soc. p. 1778.

Ingold (1921). J, chem. Soc. 119, 305, 951.

— \& Shoppee (1929). J. chem. Soc. p. 1199.

\& Wilson (1933). J. chem. Soc. p. 1493.

Karrer, Helfenstein, Wehrli \& Wettstein (1930). Helv. chim. Acta, 13, 1084.

Kuhn \& Brockmann (1931). Hoppe-Seyl. Z. 200, 255.

- $(1933,1)$. Klin. Wschr. 12, 972.

$(1933,2)$. Ber. dtsch. chem. Ges. 66, 407.

Smith \& Milner (1934). J. biol. Chem. 104, 437.

Stefan (1898). J. prakt. Chem. 58, 111.

Zechmeister \& Tuzson (1938). Biochem. J. 32, 1305. (1939). Ber. dtsch. chem. Ges. 72, 1340.

Zeitschel (1906). Ber. dtsch. chem. Ges. 39, 1780. 